Rev. Elev. Méd. vét. Pays trop., 1978, 31 (1) : 45-55.

\title{
Les leucocytes des bovins de la zone tropicale de l'Afrique de l'Ouest
}

\author{
par P. CUQ $\left(^{*}\right)$, A. J. AKAKPO $\left({ }^{*}\right)$ et D. FRIOT $(* * *)$
}

\begin{abstract}
RÉSUMÉ
Les leucocytes des bovins (taurins et zébus) du Sénégal et du Togo sont étudiés. La morphologie et la numération de chaque type cellulaire et les formules leucocytaires sont tour à tour examinées ainsi que les variations en fonction de l'espèce, de la race, de l'état sexuel, de l'âge et de la saison. Les résultats sont comparés à ceux d'autres populations bovines de l'Afrique et des régions tempérées.
\end{abstract}

Dans un précédent article nous avons rapporté les caractéristiques biologiques des érythrocytes des bovins de la zone tropicale de l'Afrique de l'Ouest. Le présent travail est consacré aux leucocytes.

\section{MATÉRIEL ET MÉTHODE}

Comme pour les érythrocytes (5), 639 taurins et zébus du Sénégal et du Togo ont été utilisés. Les mêmes prélèvements ayant servi pour l'étude des globules rouges et celle des globules blancs, ces animaux se répartissent en mêmes groupes ethniques et sexuels et en mêmes classes d'âge que dans le travail précédent. Pour la même raison, la répartition saisonnière et les techniques de prélèyement et de coloration sont les mêmes.

Les numérations ont été aussi effectuées avec les hématimètres de Malassez et de Neubauer et le numérateur automatique Picoscale. La formule de correction des valeurs lues au compteur du numérateur Picoscale a été pour les globules blancs $Y_{\mathrm{GB}}=0,00067 \mathrm{x}^{2}+9,964 \mathrm{x}+7$, dans laquelle $\mathrm{x}$ est le nombre lu au compteur et $\mathrm{Y}_{\mathrm{GB}}$ le nombre des leucocytes en valeur corrigée.

(*) Professeur, Ecole Inter-Etats des Sciences et Médecine Vétérinaires de Dakar, Rép. du Sénégal.

(**) Assistant, E. I. S. M. V., Dakar, Rép. du Sénégal.

(***) Laboratoire National de l'Elevage et de Recherche vétérinaire (I.S. R. A.) Dakar-Hann, Rép. du Sénégal.
Les mêmes contrôles de précision des mesures et de comparaison des résultats des appareils ont été effectués. Ils aboutissent aux mêmes conclusions que pour les hématies et ils nous ont conduit à traiter séparément les résultats obtenus avec les hérnatimètres et ceux fournis par le numérateur automatique.

\section{RÉSULTATS}

Les résultats se rapportent à la morphologie des leucocytes, à leurs numérations et à l'établissement des formules leucocytaires.

\section{MORPHOLOGIE DES LEUCOCYTES}

A côté des plasmocytes, toujours très rares (leur fréquence ne dépasse jamais 1 p. 100), des érythrocytes et des plaquettes sanguines, le sang des zébus et des taurins tropicaux comprend des lymphocytes, des monocytes et des granulocytes neutrophiles, éosinophiles et basophiles.

\section{A. Les lymphocytes}

La population lymphocytaire du sang des bovins de la zone tropicale de l'Afrique de l'Ouest peut, comme celle de la plupart des mammifères, être subdivisée en grands et petits lymphocytes. 


\section{1) Les grands lymphocytes}

Les grands lymphocytes sont le plus souvent arrondis ou sphériques. Leur diamètre varie de 13,5 à $16,8 \mu(14,5 \mu$ en moyenne). Leur noyau est habituellement central ou légèrement excentrique. Il est le plus souvent arrondi, avec parfois un contour légèrement encoché. Lorsqu'il subit de la part des érythrocytes le phénomène d' "attraction hémoglobinique $»$, il se déforme et s'accole à la membrane cytoplasmique. Après coloration au May Grünwald-Giemsa(M.G.-G.), le noyau présente des masses de chromatine compactes et violacées, séparées par des zones plus claires. Le nucléole est toujours très visible.

Le cytoplasme est basophile. Après coloration au M. G.-G., il prend une teinte allant du bleu franc au bleu clair. Il renferme parfois des granulations azurophiles souvent entourées d'un halo clair et localisées dans la zone où le cytoplasme est le plus abondant. Le nombre des grands lymphocytes granulés est très variable. Selon les frottis, ils peuvent être très rares ou représenter la majorité de la population.

Chez les bovins de la zone tropicale de l'Afrique de l'Ouest, il n'est pas rare de rencontrer des grands lymphocytes atypiques: lymphocytes à 2 noyaux, lymphocytes à cytoplasme vacuolisé ou lymphocytes de taille supérieure à la normale (dits lymphocytes monocytoïdes) dont les noyaux sont à chromatine lâche et le cytoplasme bleu clair et très abondant.

\section{2) Les petits lymphocytes}

Les petits lymphocytes sont arrondis. Ils mesurent 7 à $11,2 \mu(8,5 \mu$ en moyenne). Leur noyau est rond ou parfois réniforme. Il occupe presque toute la cellule et ne laisse subsister qu'un très mince liseré de cytoplasme disposé en croissant. Chez les taurins et les zébus étudiés, il n'est pas rare d'observer aussi des lymphocytes apparemment réduits à leur seul noyau.

Après coloration au M. G.-G., le cytoplasme prend une teinte bleu franc et le noyau devient violet foncé homogène. Parfois il présente des ombres (ombres de Grumprecht) qui caractérisent le noyau d'une cellule en voie de dégénérescence.

\section{B. Les monocytes}

Sur les bovins étudiés, nous avons observé 2 tailles de monocytes et quelques formes atypiques.

\section{1) Les grands monocytes}

Les grands monocytes sont arrondis, ils mesurent 15,5 à $23 \mu$ (17 $\mu$ en moyenne). Ce sont les plus grandes cellules du sang.

Leur noyau est volumineux et en position variable, centrale ou périphérique. Le plus souvent, il est réniforme ou fortement encoché ou en fer à cheval et sa chromatine est claire et ne forme pas de blocs compacts, mais nous en avons observés de plus grands, à chromatine dense et à contours irréguliers. Le nucléole est généralement invisible. Lorsqu'il subit le phénomène de l'«attraction hémoglobinique », le noyau des grands monocytes subit des modifications de forme et de coloration. En se rapprochant de la membrane cytoplasmique, il s'étire et prend des formes variées en trèfle, en papillon, en haltère ou en arc. Sa chromatine attirée vers le pôle le plus proche de la cellule chargée d'hémoglobine s'y condense et s'étire en travées d'aspect "peigné » dans la zone centrale du noyau.

Le cytoplasme, toujours plus abondant que celui des grands lymphocytes, est gris bleu, " couleur de ciel d'orage", après coloration au M. G.-G. Parfois il est plus clair, mais parsemé de très fines granulations sombres.

\section{2) Les petits monocytes}

A la taille près (10 à $15 \mu$ ), les petits monocytes ressemblent aux grands. Il est souvent difficile de les distinguer des grands lymphocytes à noyaux ronds, encochés ou étalés. Lorsqu'ils subissent le phénomène d'attraction hémoglobinique, leur noyau s'étrangle en son milieu et ils peuvent être confondus avec un lymphocyte en division. La coloration du cytoplasme en gris bleuté est alors le meilleur critère de distinction.

\section{3) Formes atypiques de monocytes}

Les formes atypiques, telles les cellules à 2 noyaux, sont rares chez les bovins tropicaux. Nous avons par contre très souvent observé des cellules à inclusions dont la nature, vraisemblablement parasitaire, n'a pas pu être exactement déterminée.

\section{Les granulocytes basophiles}

Les granulocytes basophiles des taurins et des zébus que nous avons étudiés mesurent 12 à 
$13 \mu$ de diamètre $(12,5 \mu$ en moyenne). Ce sont les plus petits granulocytes.

Le noyau, très souvent masqué par les granulations qui le recouvrent, est polylobé. Le cytoplasme est acidophile, il prend une coloration rose orangée au M. G.-G. Il contient des granulations plus ou moins basophiles dont la couleur va du rouge violacé au bleu foncé. Ces granulations dont le diamètre est toujours inférieur à $1 \mu$ sont très nombreuses. Elles occupent tout le cytoplasme et interfèrent souvent sur le noyau.

\section{Les granulocytes éosinophiles}

La taille des granulocytes éosinophiles varie de 12,5 à $14,5 \mu$. Leur noyau est dense, à chromatine en blocs. Il est souvent bi ou tri-lobé mais il n'est pas rare d'en observer d'allongés en « batonnet ». Les noyaux bilobés ont une forme de bissac. Dans les trilobés, les masses nucléaires sont alignées en chaine et disposées en arc de cercle. L' « attraction hémoglobinique » peut déformer ce noyau qui s'accole alors à la membrane cytoplasmique.

Après coloration au M. G.-G. le cytoplasme est grisâtre. Il renferme des granulations sphériques de moins de $1 \mu$ de diamètre et colorées en rouge ou rose-orangé à reflets jaunâtres. Elles sont très abondantes et réparties dans tout le cytoplasme. Sur certains frottis, les granulations se colorent parfois anormalement. Elles deviennent alors noir bleuté, très proches par leur couleur des granulations basophiles.

Sur 3 animaux nous avons observé des granulocytes éosinophiles de taille très inférieure à la normale avec un noyau à chromatine dense qui rappellent les formes décrites dans l'anomalie de Pelger-Huet chez l'homme et le lapin $(12,3)$.

\section{E. Les granulocytes neutrophiles}

Les granulocytes neutrophiles sont arrondis lorsqu'ils ne sont pas déformés par une cellule voisine ou soumis au phénomène de l' " attraction hémoglobinique $"$. Leur diamètre varie de 11,5 à $14,5 \mu$.

Dans les cellules jeunes, le noyau est incurvé ou en bissac, alors que dans les cellules plus anciennes le noyau est composé de 3 à 5 lobes (ou segments nucléaires) réunis par des ponts chromatiniens d'épaisseur variable. Les segments nucléaires sont de formes diverses: arrondie, ovalaire ou irrégulière. Ils se disposent de façon variable, en figures polymorphes qui vont du C ou du $\mathrm{S}$ à des images en $\mathrm{Y}$ ou en $\mathrm{Z}$... La chromatine est toujours dense et forme des masses foncées séparées par des zones plus claires.

Le nombre des segments nucléaires des granulocytes neutrophiles dépend de l'âge de la cellule. Il est à la base de la formule d'Arneth qui, chez le zébu normal, se traduit par la prépondérance des cellules à noyaux trilobés. L'indice de segmentation nucléaire, dans le même cas est de 3,13. Sur les bovins étudiés, nous avons observé des déviations à gauche de la formule d'Arneth avec indice de segmentation nucléaire à 1,22 lors d'action régénérative réactionnelle au cours d'anémies sévères et avec indice de segmentation nucléaire à 2,08 au cours d'une funiculite survenue après castration sur un taureau zébu. Nous n'avons pas observé de déviation à droite de la formule d'Arneth.

Le noyau des granulocytes neutrophiles du zébu porte parfois un grêle appendice dont la forme est habituellement celle d'une baguette de tambour de longueur et de diamètre variables. Cet appendice ou corps de Barr représente pour DAVIDSON et SMITH (6) la chromatine sexuelle. Il est, d'après BRIGGS (4) assez fréquent chez la femelle ( 1 à 29 fois sur 50 cellules) et absent chez le mâle qui présente parfois cependant des pseudo-corps de Barr. La différence des fréquences selon les sexes permet de poser le diagnostic du sexe génétique. Pour UNDRITZ (12), dans l'espèce humaine, il faut au moins 6 cellules à corps de Barr sur 500 pour poser le diagnostic de sexe femelle. Sur les animaux des régions tropicales de l'Afrique de l'Ouest que nous avons examinés, les femelles ont une fréquence minimale de 2 corps de Barr pour 200 granulocytes neutrophiles alors que chez le mâle leur fréquence maximale est de 1/200.

Le cytoplasme des granulocytes neutrophiles est acidophile et prend, après coloration au M. G.-G., une teinte rosée plus ou moins claire. Il contient des granulations marron ou rose selon le $\mathrm{pH}$ des liquides de lavage et de dilution. Ces granulations sont si petites qu'elles se présentent souvent sous l'aspect d'une fine poussière à peine visible.

Les formes atypiques des polynucléaires neutrophiles des bovins des régions tropicales de l'Afrique de l'Ouest sont rares. Nous avons observé quelques cellules à noyau hypersegmenté (5 à 6 lobes) et une cellule polyploïde en mitose. 
TABLEAU N ${ }^{\circ}$ I - Numérations 1eucocytarres .

\begin{tabular}{|c|c|c|c|c|}
\hline & $\begin{array}{c}\text { Moyenne générale } \\
\mathrm{N} \text { et } \tilde{\mathrm{x}} \pm \text { intervalle } \\
\text { de conflance }\end{array}$ & $\begin{array}{c}\text { Males castrés } \\
\text { N et } \bar{x} \pm \text { intervalle } \\
\text { de confiance }\end{array}$ & $\begin{array}{c}\text { Femelles } \\
\text { Net } \bar{x} \pm \text { intervalle } \\
\text { de confiance }\end{array}$ & $\begin{array}{c}\text { Males entiers } \\
\mathrm{N} \text { et } \bar{x} \pm \text { intervalie } \\
\text { de confiance }\end{array}$ \\
\hline \multicolumn{5}{|l|}{ HEMATIMETRE $\left(\mathrm{x} .10^{3}\right)$} \\
\hline Taurins du Togo & $\frac{9,03 \pm 0,57}{(104)}$ & $9,30 \pm \frac{(69)}{0,69}$ & $8,14 \pm \frac{ \pm 1,19}{(23)}$ & $9,15 \pm 2,33$ \\
\hline Zébus du Togo & $9,02 \pm 0,61$ & $9,53 \pm \frac{0,99}{(58)}$ & $7,24 \underset{(\overline{7})}{3,64}$ & $8,63 \underset{(44)}{(44)}$ \\
\hline $\begin{array}{l}\text { Zébus Gobra du } \\
\text { Sénégal }\end{array}$ & $6,86 \pm 0,066$ & $7,18 \pm 0,88$ & $6,95 \pm 1,62$ & $\begin{array}{c}5,29 \pm 0,95 \\
(\overline{1} 1)\end{array}$ \\
\hline $\begin{array}{l}\text { Zëbus Maure du } \\
\text { Sénégal }\end{array}$ & $6,60 \underset{(12)}{12}$ & & & \\
\hline PICOSCALE (X.10 $\left.0^{3}\right)$ & & & & \\
\hline Taurins du Sênégal & $6,94 \underset{(106)}{ \pm 0,55}$ & $6,90 \pm 0,59$ & $\left.6,88 \pm \frac{1}{7}\right)^{3,14}$ & $7,14 \underset{(16)}{ \pm 2,01}$ \\
\hline $\begin{array}{l}\text { Zébus Gobra dus } \\
\text { Sênégal }\end{array}$ & $\begin{array}{c}6,55 \pm 0,56 \\
(192)\end{array}$ & $6,72+0,49$ & $6,01 \pm \underset{(50)}{ \pm 0,68}$ & $6,76 \underset{(68)}{ \pm 0,53}$ \\
\hline $\begin{array}{l}\text { Zebus Maure dtu } \\
\text { Sérégal }\end{array}$ & $6,85 \pm \frac{1}{(21)}$ & & & \\
\hline
\end{tabular}

\section{NUMÉRATION LEUCOCYTAIRE}

Les numérations leucocytaires ont porté sur des taurins et des zébus du Togo et du Sénégal. Elles ont été effectuées à l'hématimètre et au numérateur automatique Picoscale.

\section{A. Moyennes des numérations leucocytaires}

Dans le tableau $\mathrm{n}^{\circ} \mathrm{I}$ les résultats sont exprimés en $1.10^{3}$ leucocytes.

\section{1) Influence de l'espèce et de la race}

Concernant le rôle de l'espèce et de la race sur la numération leucocytaire, le fait le plus caractéristique est que les taurins comme les zébus du Togo présentent des moyennes significativement plus élevées que ceux du Sénégal.

Pour les taurins, les chiffres sont respectivement $9,03 \cdot 10^{3}$ au Togo (hématimètre) et $6,94.10^{3}$ au Sénégal, (Picoscale) tous 2 très inférieurs du reste au chiffre rapporté par ODUYE et collab. (9) pour les Ndamas du Nigeria $\left(12,10 \cdot 10^{3}\right)$.

Pour les zébus, les chiffres de numération hématimétrique sont respectivement, au Togo, $9,02 \cdot 10^{3}$ et, au Sénégal, $6,60 \cdot 10^{3}$ pour la race Maure et $6,86 \cdot 10^{3}$ pour la race Gobra. ODUYE et collab. (9) estiment à $9,90 \cdot 10^{3}$ la numération leucocytaire du zébu White Fulani, VOHRADSKY (13) à $8,83 \cdot 10^{3}$ celle des Sokoto Gudali, chiffres voisins de ceux que nous avons obtenus au Togo où ces 2 races coexistent, mais très inférieurs à ceux que rapportent ADAM et collab. (1) pour le bétail du Soudan $\left(12,10.10^{3}\right)$ et SMITH (11) pour les vaches d'Ouganda $\left(11,22 \cdot 10^{3}\right)$. Selon cet auteur, le nombre élevé des leucocytes est dû à l'action de germes pathogènes, mais il est vraisemblable que les facteurs régionaux d'environnement interviennent aussi pour interférer sur les rôles éventuels de l'espèce et de la race.

\section{2) Influence du sexe}

Il n'existe aucune différence significative entre les numérations leucocytaires des divers groupes sexuels. Nous pouvons remarquer cependant que les femelles ont, dans l'ensemble, des moyennes inférieures à celles des mâles entiers et castrés.

\section{3) Influence de l'âge}

Bien qu'aucune différence significative n'ait été mise en évidence, nous avons remarqué, comme le rapportent SCHALM (10) et WYERS (14) pour "Bos taurus" dans les régions tempérées, que la numération leucocytaire diminue nettement avec l'âge chez le taurin Ndama et chez les femelles zébu de race Gobra. Pour ces dernières, dans la série traitée au Picoscale, les moyennes sont successivement $10,25 \cdot 10^{3}$; $8,81 \cdot 10^{3} ; 6,73 \cdot 10^{3} ; 6,27 \cdot 10^{3}$ et $6,02 \cdot 10^{3}$. La cause la plus fréquemment évoquée pour expliquer cette baisse est la multiparité chez les femelles. 


\section{4) Influence de la saison}

L'influence de la saison a été étudiée sur le lot de zébus Gobra testé au Picoscale sans révéler de différence significative.

\section{NUMERATIONS DES DIVERS TYPES CELLULAIRES}

\section{A. Numérations des lymphocytes}

1) Moyennes des numérations lymphocytaires

Dans le tableau $\mathrm{n}^{\circ} \mathrm{II}$, les résultats sont exprimés en $1.10^{1}$ lymphocytes.

\section{2) Discussion}

\section{a) Influence de l'espèce et de la race}

Dans les séries de numérations aux hématimètres, il n'y a pas de différence significative à 5 p. 100 entre les moyennes générales des taurins de races Lagunaires, les zébus du Togo et les zébus du Sénégal. De même, les séries Picoscale montrent qu'il n'y a pas de différence significative entre les taurins et les zébus du Sénégal.

L'espèce et la race ne semblent donc pas avoir d'influence sur la numération lymphocytaire.

b) Influence du sexe

Bien qu'aucune différence significative n'ait pu être mise en évidence à partir des effectifs que nous avons étudiés, nous remarquons que les femelles ont une nette tendance à présenter une numération lymphocytaire plus faible que celle des mâles entiers et castrés. Les valeurs sont respectivement $306,3 \cdot 10^{1}$ contre $425,6.10^{1}$ et $364,9 \cdot 10^{1}$ (hématimètre) pour les taurins du
Togo ; $219,9 \cdot 10^{1}$ contre $250,8 \cdot 10^{1}$ et $264,2.10^{1}$; (Picoscale) pour les taurins du Sénégal ; $258,1 \cdot 10^{1}$ contre $332,0 \cdot 10^{1}$ et $402,8 \cdot 10^{1}$ (hématimètre) pour les zébus du Togo et $205,6.10^{1}$ contre $273,8 \cdot 10^{1}$ et $261,5 \cdot 10^{1}$ (Picoscale) pour les zébus du Sénégal.

Comme celui des leucocytes, le nombre des lymphocytes des bovins de l'Afrique de l'Ouest que nous avons étudiés est sensiblement plus faible chez les femelles que chez les mâles.

\section{c) Influence de l'âge}

Il n'existe pas de différence significative entre les numérations lymphocytaires des différentes classes d'âge. Nous remarquons cependant que chez les zébus du Togo et du Sénégal le nombre des lymphocytes diminue avec l'âge. Le même phénomène a été observé chez les taurins des régions tempérées (14).

\section{d) Influence de la saison}

L'influence de la saison a été étudiée sur le zébu Gobra traité au Picoscale. Sur ce groupe, l'analyse de la variance montre qu'il existe une différence significative entre la fin de la saison des pluies $\left(205,3 \cdot 10^{1} \pm 23,7 \cdot 10^{1}\right)$ et mars/avril $\left(325,4 \cdot 10^{1} \pm 40,7 \cdot 10^{1}\right)$. Le niveau le plus bas est atteint en post-hivernage

$$
\left(130,5 \cdot 10^{1} \pm 19,8 \cdot 10^{1}\right) \text {. }
$$

\section{B. Numérations des monocytes}

\section{1) Moyennes des numérations de monocytes}

Dans le tableau $n^{\circ}$ III les résultats sont exprimés en $1.10^{1}$ monocytes.

TABLEAU N ${ }^{\circ}$ II - Numérations des 1ymphocytes.

\begin{tabular}{|c|c|c|c|c|}
\hline & $\begin{array}{c}\text { Moyenne génërale } \\
\mathrm{N} \text { et } \bar{x} \pm \text { intervalle } \\
\text { de confiance }\end{array}$ & $\begin{array}{c}\text { Yâles castrés } \\
\mathrm{x} \text { et } \overrightarrow{\mathrm{x}} \pm \text { intervalle } \\
\text { de confiance }\end{array}$ & $\begin{array}{c}\text { Feme } 1 \text { les } \\
\mathrm{N} \text { et } \overline{\mathrm{x}} \pm \text { Intervalle } \\
\text { de confiance }\end{array}$ & $\begin{array}{l}\text { rales entiers } \\
N \text { et } \bar{x} \pm \text { intervalle } \\
\text { de confiance }\end{array}$ \\
\hline \multicolumn{5}{|l|}{ HEMATIMETRE $\left(\mathrm{X} .10^{1}\right)$} \\
\hline Taurins du Togo & $\frac{359,0+30,5}{(\overline{1} 04)}$ & $364,9+32,3$ & $306,3 \pm 66,0$ & $425,6 \pm 154,0$ \\
\hline Zëbus du Togo & $365,0 \pm \frac{33,0}{(109)}$ & $402,8 \pm \frac{5}{(58)}$ & $258,1 \pm\left(\frac{1}{7}\right) 100,1$ & $332,0+\frac{\left(\frac{4}{4}\right)}{40,5}$ \\
\hline $\begin{array}{l}\text { Zébus Gobra du } \\
\text { Sénégal }\end{array}$ & $311,1 \pm 50,2$ & $375,1 \pm 70,3$ & $251,4 \underset{(14)}{ \pm 70,1}$ & $250,4 \underset{\left(\frac{6}{6}\right)}{ \pm} 65,6$ \\
\hline PICOSCALE $\left(\mathrm{X} .10^{\mathrm{l}}\right)$ & & & & \\
\hline Taurins du Sénëgal & $259,3 \pm 2 \frac{22,7}{(106)}$ & $\begin{array}{c}264,2 \pm 26,2 \\
(\overline{8} 3)\end{array}$ & $219,9 \pm 98,3$ & $250,8+\underset{(16)}{ } 61,8$ \\
\hline $\begin{array}{l}\text { Zébus Gobra du } \\
\text { Sërëgal }\end{array}$ & $251,4 \pm 29,5$ & $261,5 \underset{(74)}{ \pm} 32,4$ & $205,6 \pm \frac{(50)}{(50)}$ & $273,8+26,0$ \\
\hline
\end{tabular}


TABLEAU $N^{\circ} I I I$ - Numérations des monocytes.

\begin{tabular}{|c|c|c|c|c|}
\hline & $\begin{array}{c}\text { Moyenne générale } \\
\mathrm{N} \text { et } \overline{\mathrm{x}} \pm \text { incervalle } \\
\mathrm{de} \text { confiance }\end{array}$ & $\begin{array}{c}\text { Mâles castrés } \\
\mathrm{N} \text { et } \overline{\mathbf{x}} \pm \text { intervalle } \\
\text { de confiance }\end{array}$ & $\begin{array}{c}\text { Femellea } \\
N \text { et } \bar{x} \pm \text { intervalle } \\
\text { de confiance }\end{array}$ & $\begin{array}{c}\text { Mâles entiers } \\
\text { N et } \bar{x} \pm \text { intervalle } \\
\text { de confiance }\end{array}$ \\
\hline \multicolumn{5}{|l|}{ HEMATIMETRE $\left(\times 10^{1}\right)$} \\
\hline Taurins du Togo & $\frac{32,0 \pm 4,5}{(104)}$ & $32,3 \pm 5,4$ & $30,7 \pm \frac{ \pm}{(23)} 10,5$ & $32,2 \pm 15,3$ \\
\hline Zëbus du Togo & $31,1 \pm 4,3$ & $32,4 \underset{\left(\frac{5}{5} 8\right)}{5,9}$ & $25,8 \pm \underset{(\overline{7})}{23,8}$ & $\left.30,0 \pm \frac{(4}{44}\right) 6,7$ \\
\hline Zëbus du Sénégal & $47,1 \underset{(50)}{ \pm}$ & $43,9 \pm \frac{1}{(29)}$ & $51,3 \pm 15,3$ & $51,7 \underset{\left(\frac{6}{6}\right)}{26,3}$ \\
\hline \multicolumn{5}{|l|}{ PICOSCALE $\left(\mathrm{X} 10^{1}\right)$} \\
\hline Taurins du Sénëgal & $39,0 \pm 5,4$ & $\frac{39,9 \pm 6,4}{(83)}$ & $30,3 \pm\left(\frac{1}{7}\right) 13,2$ & $38,0 \pm \frac{13,8}{(16)}$ \\
\hline Zébus du SënégaI & $\begin{array}{c}35,9 \pm 5,6 \\
(\overline{1} 92)\end{array}$ & $\frac{36,7+4,6}{\left(\frac{7}{4}\right)}$ & $34,9 \pm 7,5$ & $35,6 \pm \frac{ \pm}{(68)} 5,6$ \\
\hline
\end{tabular}

\section{2) Discussion}

\section{a) Influence de l'espèce et de la race}

La numération monocytaire hématimétrique des zébus du Sénégal $\left(47,1.10^{1}\right)$ diffère significativement de celles des zébus $\left(31,1.10^{1}\right)$ et des taurins $\left(32,0,10^{1}\right)$ du Togo. La numération automatique montre en outre qu'il n'y a pas de différence significative entre taurins et zébus du Sénégal.

Alors que leurs numérations leucocytaires sont plus élevées, les bovins du Togo ont moins de monocytes que ceux du Sénégal.

\section{b) Influence du sexe}

Bien qu'il n'existe pas de différence significative à 5 p. 100 entre les divers groupes sexuels, nous remarquons chez les taurins du Togo et du Sénégal, une tendance des femelles à présenter des numérations monocytaires plus faibles que celles des mâles entiers et castrés : $30,7 \cdot 10^{1}$ contre $32,2 \cdot 10^{1}$ et $32,3 \cdot 10^{1}$ au Togo (hématimètre) et $30,3 \cdot 10^{1}$ contre $38,0 \cdot 10^{1}$ et $39,9 \cdot 10^{1}$ au Sénégal (Picoscale). Il en est de même pour les zébus : $25,8 \cdot 10^{1}$ contre $30,0 \cdot 10^{1}$ et $32,4.10^{1}$ au Togo (hématimètre) et $34,9.10^{1}$ contre $35,6 \cdot 10^{1}$ et $36,7 \cdot 10^{1}$ au Sénégal (Picoscale).

Les femelles ont donc, dans l'ensemble, tendance à posséder moins de monocytes que les mâles.

\section{c) Influence de l'âge et de la saison}

Ni l'âge ni la saison n'interviennent de façon significative dans la numération monocytaire des bovins que nous avons étudiés.

\section{Numérations des granulocytes neutrophiles}

1) Moyennes des numérations des granulocytes neutrophiles

TABLEAU $N^{D}$ IV - Numërations de granulocytes neutrophiles.

\begin{tabular}{|c|c|c|c|c|}
\hline & $\begin{array}{c}\text { Moyenne gënérale } \\
\text { Net } \bar{x} \pm \text { intervalle } \\
\text { de confiance }\end{array}$ & $\begin{array}{c}\text { Mâles castrés } \\
\mathrm{N} \text { et } \overline{\mathrm{X}} \pm \text { intervalle } \\
\text { de confiance }\end{array}$ & $\begin{array}{l}\mathrm{N} \text { et } \overline{\mathrm{x}} \pm \text { interva11e } \\
\text { de confiance }\end{array}$ & $\begin{array}{c}\text { Mâles entiers } \\
N \text { et } \bar{x} \pm \text { intervalle } \\
\text { de confiance }\end{array}$ \\
\hline \multicolumn{5}{|l|}{ HEPHATIMETRE (X.10 $\left.{ }^{1}\right)$} \\
\hline Taurins du logo & $\frac{429,0 \pm 36,5}{(104)}$ & $448,7 \pm \frac{ \pm}{(59)} 48,9$ & $400,4 \underbrace{}_{(23)} 61,5$ & $370,6 \pm \frac{(\overline{1} 2)}{98,9}$ \\
\hline Zêbus du Togo & $\frac{469,6 \pm 43,4}{(109)}$ & $484,4 \pm \frac{ \pm}{(58)} 65,0$ & $411,0+272,8$ & $459,5 \underset{(44)}{58,3}$ \\
\hline Zébus du Sénégal & $355,7 \underset{(50)}{ \pm}$ & $344,9 \pm 123,8$ & $401,4 \underset{(15)}{ \pm 136,0}$ & $292,9+130,0$ \\
\hline \multicolumn{5}{|l|}{ I'ICOSCALE $\left(X \cdot 10^{1}\right)$} \\
\hline $\begin{array}{l}\text { Taurins idama du } \\
\text { Senêggal }\end{array}$ & $\begin{array}{c}316,9+32,3 \\
(106)\end{array}$ & $306,4+\frac{ \pm}{(83)} 32,6$ & $366,8 \pm 166,4$ & $349,2 \pm 129,6$ \\
\hline $\begin{array}{l}\text { ¿êbus Gobra ău } \\
\text { Sènéga1 }\end{array}$ & $\begin{array}{c}310,8+41,8 \\
(192)\end{array}$ & $309,8 \pm \frac{(74)}{36,2}$ & $307,3+\frac{ \pm}{(50)} 40,6$ & $314,3 \pm\left(\frac{ \pm}{68}\right) 36,0$ \\
\hline
\end{tabular}


Dans le tableau $\mathrm{n}^{\circ} \mathrm{IV}$, les 1ésultats sont exprimés en $1.10^{1}$ granulocytes neutrophiles

\section{a) Influence de l'espèce et de la race}

Sans qu'il existe de différences significatives à 5 p. 100 par la méthode d'analyse de la variance, on remarque que les bovins du Togo ont une numération neutro-granulocytaire plus élevée que ceux du Sénégal. Comme pour la numération leucocytaire, les infections latentes et les conditions d'environnement pourraient, au moins en partie, être évoquées pour expliquer ces différences.

\section{b) Influence du sexe}

Le sexe ne semble pas avoir d'influence nette sur la numération des granulocytes neutrophiles des bovins de l'Afrique tropicale de l'Ouest que nous avons étudiés. Aucune différence significative n'a pu être mise en évidence et les variations que nous pouvons observer intéressent tantôt les mâles entiers (taurins du Togo et zébus Gobra traités à l'hématimètre) tantôt les mâles castrés (taurins Ndama), et tantôt les femelles (zébus du Togo et zébus Gobra traités au Picoscale).

\section{c) Influence de l'âge}

Chez les taurins des régions tempérées, la plupart des auteurs dont WYERS (14) et MOBERG (8) estiment que le nombre des polynucléaires neutrophiles diminue avec l'âge. Bien qu'aucune différence significative à 5 p. 100 n'ait été mise en évidence, la même tendance s'observe sur les femelles des taurins du Togo, et sur les femelles et les mâles castrés des zébus du Sénégal.

\section{d) Influence de la saison}

Aucune différence significative révélatrice d'une influence de la saison n'a été mise en évidence. Nous pouvons observer cependant que sur le lot des zébus Gobra traité au Picoscale, le taux le plus élevé est atteint, comme pour les lymphocytes à la période de «printemps » (mars-avril).

\section{Numérations des granulocytes éosinophiles}

1) Moyennes des numérations des granulocytes éosinophiles

Dans le tableau $n^{\circ} \mathrm{V}$ les résultats sont exprimés en $1.10^{1}$ granulocytes éosinophiles.

2) Discussion

\section{a) Influence de l'espèce et de la race}

Les 3 lots traités à l'hématimètre révèlent une différence significative entre zébus et taurins du Togo $\left(36,8 \cdot 10^{1}\right.$ et $\left.76,5 \cdot 10^{1}\right)$. De même pour les 2 lots traités au Picoscale, il existe une différence significative entre zébus et taurins du Sénégal $\left(53,0.10^{1}\right.$ et $\left.77,8 \cdot 10^{1}\right)$. Les taurins ont donc un nombre plus élevé de granulocytes éosinophiles que les zébus. Il n'est pas certain cependant que cette différence soit due à l'espèce. Il convient en effet de tenir compte de la présence éventuelle d'endoparasites dont l'infestation est favorisée par l'humidité. Les taurins trypano-tolérants séjournent toute l'année dans des régions très humides. Ils sont donc normalement plus infestés que les zébus qui ne quittent pas la zone tropicale sèche et, par conséquent, sont plus facilement atteints d'éosinophilie parasitaire.

TABLEAU $\mathrm{N}^{\bullet} \mathrm{V}$ - Numërations des granulocytes eosinophiles.

\begin{tabular}{|c|c|c|c|c|}
\hline & $\begin{array}{c}\text { Moyenne générale } \\
\mathrm{N} \text { et } x+1 \text { ntervalle } \\
\text { de cuarfiance }\end{array}$ & $\begin{array}{c}\text { Yâles cas trés } \\
\therefore \text { et } \bar{x} \pm 2 n \text { tervalle } \\
\text { de confiance }\end{array}$ & $\begin{array}{c}\text { Femelles } \\
\text { iN et } \pm \text { intervalie } \\
\text { de confiance }\end{array}$ & $\begin{array}{c}\text { Mâles entiers } \\
\mathrm{N} \text { et } \mathrm{x} \pm \text { intervalie } \\
\text { de confiance }\end{array}$ \\
\hline \multicolumn{5}{|l|}{ HEMATIMETRE (X.10 $\left.{ }^{1}\right)$} \\
\hline Taurins du Togo & $\begin{array}{c}76,5 \pm 14,9 \\
(\overline{1} 04)\end{array}$ & $84,3 \pm \frac{169)}{(19,6}$ & $48,1 \pm \pm_{(23)}^{21,2}$ & $85,2 \pm{ }_{(12)}^{51,4}$ \\
\hline Zébus du Togo & $36,8 \pm 8,3$ & $34,4 \pm 12,6$ & 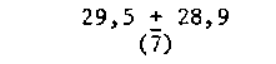 & $41,0 \underset{(44)}{ \pm}$ \\
\hline Zébus du Sênểgal & $37,4 \underset{(50)}{ \pm} 10,3$ & $39,2 \pm \underset{(29)}{ \pm 14,7}$ & $33,2 \pm 16,4$ & $38,8 \pm 4\left(\frac{6}{6}\right), 0$ \\
\hline \multicolumn{5}{|l|}{$\operatorname{PICOSCALE}\left(\mathrm{X}, 10^{1}\right.$ ) } \\
\hline $\begin{array}{l}\text { Tauring Ndama du } \\
\text { Sénégal }\end{array}$ & $\begin{array}{c}77,8 \pm 11,6 \\
(106)\end{array}$ & $79,0 \underset{(\overline{8} 3)}{ \pm 12,1}$ & $70,0 \pm\left(\frac{7}{7}\right) 5$ & $74,9 \pm 44,0$ \\
\hline $\begin{array}{l}\text { Zébus Gobra du } \\
\text { Sénégal }\end{array}$ & $53,0 \pm \underset{(192)}{ \pm 12,4}$ & $56,8+\frac{(74)}{13,1}$ & $48,8+\frac{(50)}{(2,6}$ & $52,0 \underset{(68)}{\left(\frac{1}{6}\right)}$ \\
\hline
\end{tabular}




\section{b) Influence du sexe}

Bien qu'aucune différence significative ne puisse être mise en évidence sur les lots que nous avons étudiés, il est remarquable de constater que, dans tous les cas, les femelles ont un nombre de granulocytes éosinophiles nettement plus faible que les mâles castrés et entiers. Cette particularité, pourtant hautement probable en raison de sa constance contredit LAMMERTYN (7) qui estime que chez les mammifères domestiques, il n'existe pas de différence numérique entre les populations de granulocytes éosinophiles du mâle et de la femelle.

\section{c) Influence de l'âge}

Chez les zébus Gobra mâles castrés, il existe une différence significative entre les animaux les plus jeunes ( 2 ans à 2 ans $1 / 2$ ) et ceux qui sont âgés de 6,5 à 10 ans. Les premiers ont un nombre plus faible de granulocytes éosinophiles. En outre, et bien qu'aucune différence significative ne puisse être mise en évidence, nous avons remarqué que le nombre des granulocytes éosinophiles augmente avec l'âge chez les zébus mâles entiers du Togo et du Sénégal et chez les zébus mâles castrés de race Gobra du Sénégal.

Cette observation est en accord avec les faits rapportés par de nombreux auteurs $(10,14,7)$ pour les bovins d'Europe dans lesquels le nombre des leucocytes et des monocytes diminue avec l'âge tandis que celui des granulocytes éosinophiles augmente. Il est probable que pour les taurins tropicaux l'infestation parasitaire chronique masque en partie le phénomène.

\section{d) Influence de la saison}

Sur le lot des zébus Gobra traités au numérateur automatique, il existe une différence significative entre la saison sèche où le nombre des granulocytes éosinophiles est bas $\left(39,2.10^{1}\right)$ et la saison des pluies où il est le plus élevé $\left(63,9,10^{1}\right)$. Ce phénomène doit vraisemblablement être aussi rapporté à l'infestation parasitaire de la saison humide.

\section{E. Granulocytes basophiles}

1) Moyennes des numérations de granulocytes basophiles

Dans le tableau $n^{\circ}$ VI, les résultats sont exprimés en $1.10^{1}$ granulocytes basophiles.

\section{2) Discussion}

Toutes les différences significatives que nous avons relevées tiennent au fait que certaines classes d'animaux ont des moyennes égales à 0,0 alors que d'autres, peu nombreuses, ont une moyenne supérieure à ce chiffre. L'examen attentif montre en outre de façon constante que les classes à moyenne non nulle sont composées d'une majorité de sujets dépourvus de granulocytes basophiles ou n'en possédant que quelques-uns et d'animaux très peu nombreux à basophilie accusée. Les différences mathématiques ne correspondent donc pas à des caractéristiques biologiques de groupes. Nous pouvons donc conclure que les bovins des régions tropicales de l'Afrique de l'Ouest n'ont pas ou ont très peu de granulocytes basophiles.

TABLEAU $\mathrm{N}^{\circ}$ VI - Numérations des granulocytes basophiles.

\begin{tabular}{|c|c|c|c|c|}
\hline & $\begin{array}{c}\text { Moyenne gênêrale } \\
\mathrm{N} \text { et } \mathrm{x} \pm \text { Intervalle } \\
\text { de conf iance }\end{array}$ & $\begin{array}{c}\text { Mâles castrës } \\
N \text { et } x \pm \text { intervalle } \\
\text { de confiance }\end{array}$ & $\mathrm{N}$ et $\begin{array}{l}\text { Eemelles } \\
\mathrm{de} \\
\text { de } \text { confintervalle }\end{array}$ & $\begin{array}{c}\text { Mâles entiers } \\
\mathrm{N} \text { et } \mathrm{x} \pm \text { intervalle } \\
\text { de confiance }\end{array}$ \\
\hline \multicolumn{5}{|l|}{ HEMATIMETRE (X.10 $10^{1}$ ) } \\
\hline Taurins du Togo & $\frac{0,6 \pm 0,6}{(104)}$ & $0,5+0,7$ & $0,3 \pm \underset{(23)}{0,7}$ & $1,5 \pm(12), 4$ \\
\hline Zébus du Togo & $\begin{array}{c}0,1 \pm 0,2 \\
(109)\end{array}$ & ${ }_{(58)}^{0,0}$ & 0,0 & $0,2 \pm \frac{0,5}{(44)}$ \\
\hline Zẻbus du Sénégal & $0, \frac{0,9 \pm 0,0}{(50)}$ & $0,7 \pm 0,9$ & $1,6 \pm 2,6$ & 0,0 \\
\hline \multicolumn{5}{|l|}{ PICOSCALE (X.10 $\left.10^{1}\right)$} \\
\hline $\begin{array}{l}\text { Taurins Ndama du } \\
\text { Sënëgal }\end{array}$ & $\underset{(106)}{1,0 \pm 0,5}$ & $0,9 \pm 0,6$ & $1,1_{(\overline{7})}+1,9$ & $1,3 \pm 1,2$ \\
\hline $\begin{array}{l}\text { Zébus Gobra du } \\
\text { Sénégal }\end{array}$ & $\frac{0,5 \pm 1,4}{(192)}$ & $0,4 \pm 0,4$ & $0,5 \pm 0,4$ & $0,6 \pm \frac{ \pm}{(67)}$ \\
\hline
\end{tabular}


TABLEAU $N^{\circ}$ VII - Formules sanguines.

\begin{tabular}{|c|c|c|c|c|c|c|c|c|c|c|c|c|c|}
\hline \multirow[b]{3}{*}{ TAURINS DU TOGO } & \multicolumn{6}{|c|}{ Num érations } & \multicolumn{7}{|c|}{ F O r m u 1 e $s$} \\
\hline & $\mathrm{L}$ & M & N & E & B & $\mathrm{T}$ & $\mathrm{L}$ & M & $\mathrm{N}$ & E & B & AGR & GR \\
\hline & & & & & & & & & & & & & \\
\hline Moyenne générale & 3590 & 320 & 4290 & 765 & 6 & 8981 & 0.40 & 0.04 & 0.48 & 0.08 & 0.00 & 0.44 & 0.56 \\
\hline Mâles castrës & 3649 & 323 & 4487 & 843 & 5 & 9307 & 0.39 & 0.04 & 0.48 & 0.09 & 0.00 & 0.43 & 0.57 \\
\hline Feme1les & 3063 & 307 & 4004 & 481 & 3 & 7818 & 0.39 & 0.04 & 0.51 & 0.06 & 0.00 & 0.43 & 0.57 \\
\hline Mâles entiers & 4256 & 322 & 3706 & 852 & 15 & 9151 & 0.47 & 0.04 & 0.40 & 0.09 & 0.00 & 0.51 & 0.49 \\
\hline $\begin{array}{l}\text { TAURINS DU } \\
\text { SENEGAL }\end{array}$ & & & & & & & & & & & & & \\
\hline Moyenne génêrale & 2593 & 390 & 3169 & 778 & 10 & 6940 & 0.37 & 0.06 & 0.46 & 0.11 & 0.00 & 0.43 & 0.57 \\
\hline Mâles castrés & 2642 & 399 & 3064 & 790 & 9 & 6904 & 0.38 & 0.06 & 0.44 & 0.12 & 0.00 & 0.44 & 0.56 \\
\hline Feme 11 es & 2199 & 303 & 3668 & 700 & 11 & 6901 & 0.32 & 0.04 & 0.54 & 0.10 & $0 . \infty$ & 0.36 & 0.64 \\
\hline Mâles entiers & 2508 & 380 & 3492 & 749 & 13 & 7142 & 0.35 & 0.05 & 0.49 & 0.11 & 0.00 & 0.40 & 0.60 \\
\hline ZEBUS DU TOGO & & & & & & & & & & & & & \\
\hline Moyenne génërale & 3650 & 311 & 4696 & 368 & 1 & 9026 & 0.41 & 0.03 & 0.52 & 0.04 & 0.00 & 0.44 & 0.56 \\
\hline Mâles castrĕs & 4028 & 324 & 4844 & 344 & 0 & 9540 & 0.42 & 0.03 & 0.51 & 0.04 & 0.00 & 0.45 & 0.55 \\
\hline Femelles & 2581 & 258 & 4111 & 295 & 0 & 7245 & 0.35 & 0.04 & 0.57 & 0.04 & 0.00 & 0.39 & 0.61 \\
\hline Males entiers & 3320 & 300 & 4595 & 410 & 2 & 8627 & 0.39 & 0.03 & 0.53 & 0.05 & 0.00 & 0.42 & $0.5 B$ \\
\hline $\begin{array}{l}\text { ZEBUS GOBRA DU } \\
\text { SENEGAL }\end{array}$ & & & & & & & & & & & & & \\
\hline Moyenne génêrale & 2514 & 359 & 3108 & 530 & 5 & 6516 & 0.39 & 0.05 & 0.48 & 0.08 & 0.00 & 0.44 & 0.56 \\
\hline Mâles castrés & 2615 & 367 & 3098 & 568 & 4 & 6652 & 0.39 & 0.06 & 0.47 & 0.08 & 0.00 & 0.45 & 0.55 \\
\hline Feme1les & 2056 & 349 & 3073 & 488 & 5 & 5971 & 0.34 & 0.06 & 0.52 & 0.08 & 0.00 & 0.40 & 0.60 \\
\hline Mâles entiers & 2738 & 356 & $\begin{array}{ll}3 & 143\end{array}$ & 520 & 6 & 6763 & 0.41 & 0.05 & 0.46 & 0.08 & 0.00 & 0.46 & 0.54 \\
\hline
\end{tabular}

$\mathrm{L}=$ Lymphocytes ; $\mathrm{M}=$ Monocytes ; $\mathrm{N}=$ Granulocytes neutrophiles ; $\mathrm{E}=$ Granulocytes eosinophiles ;

$B=$ Granulocy tes basophiles $; A G R=$ Agranulocytes ; $G R=$ Granulocytes .

\section{FORMULES LEUCOCYTAIRES}

\section{A. Valeurs moyennes des formules sanguines}

Le tableau $n^{\circ}$ VII rapporte les valeurs absolues et les fréquences de chaque catégorie cellulaire pour les comptages effectués sur les échantillons les plus nombreux, c'est-à-dire pour les numérations hématimétriques, des taurins (104 sujets) et des zébus (109 sujets) du Togo et pour les numérations automatiques des taurins (106 sujets) et des zébus (192 sujets) du Sénégal.

\section{B. Discussion}

\section{1) Influence de l'espèce et de la race}

L'espèce et la race n'ont aucune influence sur les proportions relatives des granulocytes et des agranulocytes. Les taurins du Togo, les zébus du Togo et les zébus du Sénégal présentent les mêmes fréquences $(56$ p. 100 de granulocytes et
44 p. 100 d'agranulocytes) non significativement différentes de celles des taurins Ndama du Sénégal (57 p. 100 et 43 p. 100). De même, les différences de fréquences entre chaque catégorie cellulaire ne présentent aucune différence significative à 5 p. 100 après analyse statistique par le " test de l'écart réduit ».

\section{2) Influence du sexe}

Bien qu'il n'existe aucune différence significative entre les fréquences globulaires relatives aux différents états sexuels des $\mathbf{4}$ groupes bovins de l'Afrique de l'Ouest que nous avons étudiés, il est intéressant de remarquer que les femelles ont un taux de granulocytes plus élevé que celui des mâles castrés et entiers chez les taurins du Sénégal ( 64 p. 100 contre 56 p. 100 et 60 p. 100), les zébus du Togo $(61$ p. 100 contre 55 p. 100 et 58 p. 100) et les zébus du Sénégal ( 60 p. 100 contre 55 p. 100 et 54 p. 100). Dans les 3 cas, 
TABLEAU $N^{\bullet}$ VIII

\begin{tabular}{|c|c|c|c|c|c|c|}
\hline \multirow[b]{2}{*}{ Numérations } & \multicolumn{4}{|c|}{ Hé ma t i mè $t r e$} & \multicolumn{2}{|c|}{ Numërateur automatique } \\
\hline & $\begin{array}{l}\text { Taurins } \\
\text { Régions } \\
\text { terpérées }\end{array}$ & $\begin{array}{l}\text { Taurins } \\
\text { Togo }\end{array}$ & $\begin{array}{l}\text { Zëbus } \\
\text { Togo }\end{array}$ & $\begin{array}{l}\text { Zébus } \\
\text { Sénéga1 }\end{array}$ & $\begin{array}{l}\text { Taurins } \\
\text { Sénéga I }\end{array}$ & $\begin{array}{c}\text { Zébus } \\
\text { Sénéga } 1\end{array}$ \\
\hline $\begin{array}{l}\text { Leucocytes } \\
\left(\mathrm{x} \cdot 10^{3}\right)\end{array}$ & $8,10^{3}$ & $9,03 \cdot 10^{3}$ & $9,02 \cdot 10^{3}$ & $\begin{array}{l}\text { G. } 6,86 \cdot 10^{3} \\
\text { M. } 6,60 \cdot 10^{3}\end{array}$ & $6,94 \cdot 10^{6}$ & $\begin{array}{l}\text { G. } 6,55 \cdot 10^{3} \\
\text { M. } 6,85 \cdot 10^{3}\end{array}$ \\
\hline $\begin{array}{l}\text { Lymphocytes } \\
\left(x \cdot 10^{1}\right)\end{array}$ & $450.20^{1}$ & $359,0.10^{1}$ & $365,0.10^{1}$ & $311,1 \cdot 10^{1}$ & $259,3.10^{1}$ & $251,4 \cdot 10^{1}$ \\
\hline $\begin{array}{l}\text { Monocytes } \\
\left(\mathrm{x} \cdot 10^{1}\right)\end{array}$ & $40.10^{I}$ & $32,0.10^{1}$ & $31,1.10^{1}$ & $47,1 \cdot 10^{1}$ & $39,0 \cdot 10^{1}$ & $35,9 \cdot 10^{1}$ \\
\hline $\begin{array}{l}\text { Granulocy tes } \\
\text { Neutrophiles } \\
\left(\mathrm{x} \cdot 10^{\mathrm{l}}\right)\end{array}$ & $200 \cdot 10^{1}$ & $429,0.10^{1}$ & $469,6 \cdot 10^{1}$ & $355,7 \cdot 10^{1}$ & $316,9 \cdot 10^{1}$ & $310,8 \cdot 10^{1}$ \\
\hline $\begin{array}{l}\text { Granulocy tes } \\
\text { Eosinophiles } \\
\left(x \cdot 10^{l}\right)\end{array}$ & $70.10^{1}$ & $76,5.10^{1}$ & $36,8 \cdot 10^{1}$ & $37,4 \cdot 10^{1}$ & $77,8.10^{1}$ & $53,0.10^{1}$ \\
\hline $\begin{array}{l}\text { Granulocytes } \\
\text { Bas ophiles } \\
\left(x .10^{1}\right)\end{array}$ & $5.10^{\mathrm{I}}$ & $0,6 \cdot 10^{1}$ & $0,1.10^{1}$ & $0,9 \cdot 10^{1}$ & $1,0.10^{1}$ & $0,5.10^{1}$ \\
\hline
\end{tabular}

cette particularité se traduit dans la formule sanguine par un accroissement de la fréquence des granulocytes neutrophiles et une baisse des lymphocytes, les autres groupes globulaires conservant à peu près les mêmes fréquences. Traduites en valeurs absolues, ces mêmes particularités correspondent à une diminution du nombre des lymphocytes, accompagnée, seulement chez les taurins du Sénégal, d'un accroissement sensible du nombre des polynuclćaires neutrophiles.

\section{CONCLUSIONS}

1) Morphologiquement, les leucocytes des taurins et des zébus du Togo et du Sénégal sont semblables à ceux des bovins des régions tempérées.
2) Les numérations que nous avons effectuées peuvent être résumées par le tableau VIII dans lequel sont reportées à titre comparatif les valeurs moyennes publiées par SCHALM en 1971 pour les taurins des régions tempérées.

3) Pour les formules sanguines les principaux résultats sont indiqués dans le tableau IX.

4) L'influence de l'espèce et de la race semble importante pour les bovins des régions tempérées dont les constantes leucocytaires diffèrent de celles des bovins des régions tropicales par le taux élevé des agranulocytes (62 p. 100 contre 43 à 44 p. 100) surtout celui des lymphocytes (58 p. 100 contre 37 à 40 p. 100 ), par le nombre réduit des granulocytes neutrophiles $\left(200.10^{1}\right.$ contre 311 à $470.10^{1}$ ) et par l'importance relative de la population granulocytaire basophile $\left(5 \cdot 10^{1}\right.$ contre 0,1 à $\left.1,0 \cdot 10^{1}\right)$.

TAELEAU N ${ }^{\circ}$ IX

\begin{tabular}{|c|c|c|c|c|c|c|c|}
\hline & $\begin{array}{c}\text { G R } \\
(\mathrm{p}, 100)\end{array}$ & $\begin{array}{c}\text { A G R } \\
(\mathrm{p} .100)\end{array}$ & $\begin{array}{c}\mathrm{I} \\
(\mathrm{p} .100)\end{array}$ & $\begin{array}{c}M \\
(p .100)\end{array}$ & $\stackrel{\mathrm{N}}{(p .100)}$ & $\begin{array}{c}E \\
(p .100)\end{array}$ & $\begin{array}{c}\mathrm{B} \\
(\mathrm{p} \cdot 100)\end{array}$ \\
\hline $\begin{array}{l}\text { Taurins-Régions } \\
\text { tempêrées } \\
\text { (Schalm, 1971) }\end{array}$ & 38 & 62 & 58 & 4 & $2 B, 5$ & 9 & 0,5 \\
\hline Taurins du Togo & 56 & 44 & 40 & 4 & 48 & 8 & 0 \\
\hline Taurins du Sénégal & 57 & 43 & 37 & 6 & 46 & 11 & 0 \\
\hline Zébus du Togo & 56 & 44 & 41 & 3 & 52 & 4 & 0 \\
\hline Zébus du Sénêgal & 56 & 44 & 39 & 5 & 48 & 8 & 0 \\
\hline
\end{tabular}


Il est probable que des facteurs infectieux et d'environnement interviennent aussi dans le déterminisme de ces particularités, mais ils apparaissent moins nettement que dans les taurins et les zébus tropicaux. Dans l'état actuel de nos connaissances, le nombre sensiblement plus élevé des leucocytes et des granulocytes neutrophiles et celui, plus faible, des monocytes chez les bovins du Togo peuvent leur être attribués. De même, si les granulocytes éosinophiles sont plus nombreux chez les taurins, la permanence de l'infestation parasitaire dans les régions tropicales humides semble en être la cause.

5) L'influence du sexe se manifeste chez les femelles par une diminution du nombre des lymphocytes, des monocytes et des granulocytes éosinophiles qui s'accompagne d'une réduction parallèle du nombre total des leucocytes et, chez les vaches taurins Ndama, zébus Peulh du
Sahel et zébus Gobra, par une fréquence plus élevée des granulocytes.

6) L'influence de l'âge se traduit par une diminution progressive du nombre des leucocytes particulièrement nette chez le taurin Ndama et la femelle zébu Gobra. Les lymphocytes diminuent aussi progressivement avec l'âge chez les zébus. Il en est de même pour les granulocytes neutrophiles chez les taurins du Togo et chez les zébus du Sénégal, femelles et mâles castrés. A l'inverse, le nombre des granulocytes éosinophiles augmente avec l'âge chez les zébus.

7) Les lymphocytes et les granulocytes neutrophiles du zébu Gobra sont plus nombreux en mars/avril, (printemps) que durant tout le reste de l'année. L'augmentation du nombre des éosinophiles que l'on constate dans cette espèce à la saison des pluies est contemporaine d'une forte infestation parasitaire.

\section{SUMMARY}

\section{Bovine leukacytes in tropical West Africa}

Bovine leukocytes (cattle, zebu cattle) were studied in Senegal and Togo. Morphology counting of each cell type and leukocytic formularies are successively reviewed as well as the variations according to the species, the breed, the sexual cycle, the age and the season. These results are compared with those from other bovine populations of Africa and temperate zones.

\section{RESUMEN}

\section{Los leucocitos de los bovinos de la zona tropical de Africa del Oeste}

Se estudian los leucocitos de los bovinos (Bos taurus y Bos indicus) de Senegal y de Togo. Alternativamente se examinan la morfologia y la numeración de cada tipo celular y las formulas leucocitarias, asi como las variaciones en función de la especie, la raza, el estado sexual, la edad y la estación. Se comparan los resultados con los de otras poblaciones bovinas de Africa y de las regiones templadas.

\section{BIBLIOGRAPHIE}

1. ADAM (S. E.I.), OBEID (H. M.) et TARTOUR (G). Serum enzyme activities and diseased ruminants in the Sudan. Acta Vet., Brno, 1974, 43:225-235.

2. AKAKPO (A. J. B.). Contribution à l'étude de l'hématologie des bovins de l'Afrıque de l'Ouest. Thèse Doct. vét., Dakar, 1976, no 14.

3. BESSIS (M.). Cellules du sang normal et pathologique. Paris, Masson et Cie, 1972.

4. BRIGGS (D. K.). The individuality of nuclear chromatin with particular reference to polymorphonuclear neutrophil leucocytes. Blood, 1958, 13 :986.

5. CUQ (P.), AKAKPO (A. J.) et FRIOT (D.), Caractères biologiques des hématies de bovıns de la zone tropicale de l'Afrique de l'Ouest. Rev. Elev. Méd. vét. Pays trop., 1977, 30 (3) : 281-292.

6. DAVIDSON (W. M.) et SMITH (D. R.). A morphological sex difference in the polymorphonuclear neutrophil leucocytes. Brit. med. J., 1954, 2 : 6.

7. LAMMERTYN (M.). L'éosınophilie parasitaire chez les mammifères. Thèse Doct. vét., Alfort, 1972, $\mathrm{n}^{\mathrm{n}} 31$.
8. MOBERG (R.). The White blood picture in sexually nature female cattle, with special references to sexual conditions. A clinical experimental study. Thesis, Stockholm, Sweden, 1955.

9. ODUYE (O. O.) et OKUNAIYE (O. A.). Etudes hematologiques chez les races bovmes White Fulani et Ndama. Bull. Epizost. Dis. Afr., 1971, 19:213-219.

10. SCHALM (O. W.). Veterinary hematology. Philadelphia, Lee and Febiger, 1971.

11. SMITH (I. M.). The blood picture of normal zebu cows in Uganda. Brit. vet. J., 1959, $115: 89-96$.

12. UNDRITZ. Planches d'hématologie. Sandoz, 1972.

13. VOHRADSKY (F.). Variations diurnes de la formule sanguine des vaches Shorthorn Ouest Africain, Ndama, Sokoto Gudali au Ghana. Acta Vet., Brno, $1971,40: 387-395$.

14. WYERS (M.). Normes hématologiques des animaux domest1ques. Bull. Ass. Fr. Vet. Microbiol. Immunol. Spec. Mal. Infect., 1973 (12) : 51-67. 